\title{
Design of Web-based Microscopic Traffic Simulator Using Silverlight Technology
}

\author{
Can Chen ${ }^{1, a}$ and Guishan Tan ${ }^{1, b}$ \\ ${ }^{1}$ School of Transportation Engineering, Tongji University, Shanghai 201804, China. \\ acanold@163.com, b364744382@qq.com
}

Keywords: Microscopic Traffic Simulator; Silverlight technology; Web GIS.

\begin{abstract}
Based on the study of Web GIS and Silverlight technology, a Web-based microscopic traffic simulator was designed. It can not only dynamically display the changes of vehicles' status but also output the simulation results for further research and analysis of the traffic operation. The design framework and the simulation process were introduced in detail. A case study on a cross of Beijing is presented. There are two main innovative points, one is that it is easy for users to create the large scale of road network with the help of Web GIS, the other is that it combines the Silverlight technology and microscopic traffic flow models, giving a new approach to realize Web-based traffic simulation at micro level, broadening the application field of Web GIS.
\end{abstract}

\section{Introduction}

With the rapid development of economy, the traffic congestion is becoming more and more serious in big cities. The traffic congestion problem is not easy to be solved because the entire traffic system is complex and stochastic. To analyze and manage the traffic effectively, many big cities have established their own microscopic simulation models of road network. The simulation models are mainly built by classical simulation softwares (e.g. VISSIM, CORSIM, PARAMICS, et al.) $[1,2]$ which have powerful functions but are complex and hard to create the large scale of road network. To make the traffic simulation get further improved and widely used, the traffic simulation should become easier to establish, access and use for users.

There are many studies about web based simulation $[3,4]$ abroad but less are related to traffic. The Web-based microscopic traffic simulator developed in this paper is combined with the Web GIS technology and realized by the Silverlight plug-in. With the help of Web GIS, it is easy to create the large scale of road network for users. The designed microscopic traffic simulator can not only display the movements of vehicles but also analyze the operation of traffic.

\section{Background}

Web GIS (Geographic Information Systems) [5] service systems which combine the GIS and Web technologies provide the various GIS services of analyzing and displaying the spatial data with friendly user-interface. It overcomes the disadvantages of traditional desktop GIS system, for it is easier to integrate with other service information of Web.

As a main technology of Rich Internet Application (RIA) [6], Silverlight inherits the excellent properties of HTML, and adopts new description mechanism-XMAL. It can develop a user interface application with richer content and more experience.

Studies about microscopic traffic simulation is carried out earlier abroad, compared with the China. There are many classical traffic simulation softwares developed by foreign campus or company and have been widely used by traffic workers. But these softwares are mostly belong to desktop system and can't be applied to China directly, unless the parameters of traffic simulation models are modified. Because the most simulation system abroad only considers the vehicle flow, while the most cities of China are mixed traffic. 


\section{System Design}

With the help of the Silverlight plug-in and Web GIS technology, the fundamental of developing web-based microscopic traffic simulator are shown in Figure 1.

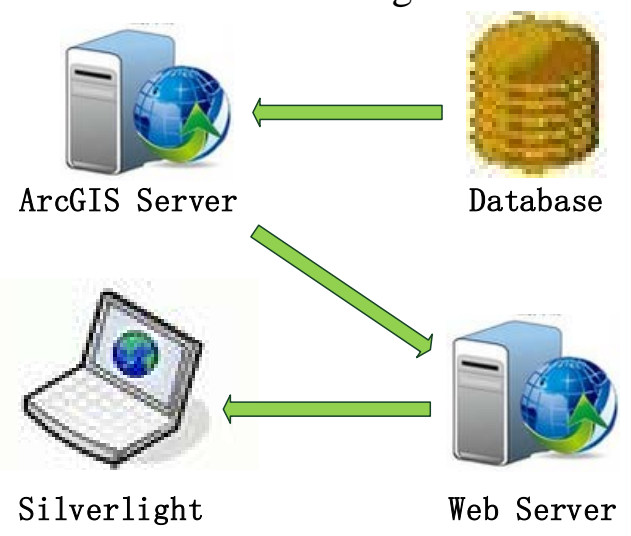

Fig 1. The fundamental of web-based microscopic traffic simulator

On the whole, the web service of GIS should be released on the ArcGIS [7] Server and the service can be used through ArcGIS API for Silverlight for client side. The database has huge and different kinds of data essential for traffic simulator, including road spatial relationship, road properties and so on. These geographic information data can be obtained by ArcGIS Server and it can transit them through Web Server. By using the Silverlight plug-in supplied by ArcGIS Server, the road network can be drawn and shown perfectly for users. It is convenient to establish traffic simulation. With the help of ArcGIS API 3.1 for Silverlight and Oracle [8] database, the microscopic traffic simulator introduced in this paper is built on the ArcGIS 10 and Silverlight 5 platforms.

The concrete system framework is shown in Figure 2. The microscopic traffic simulator includes the following four core modules: input module, output module, display and vehicle simulation modules, described in detail below.

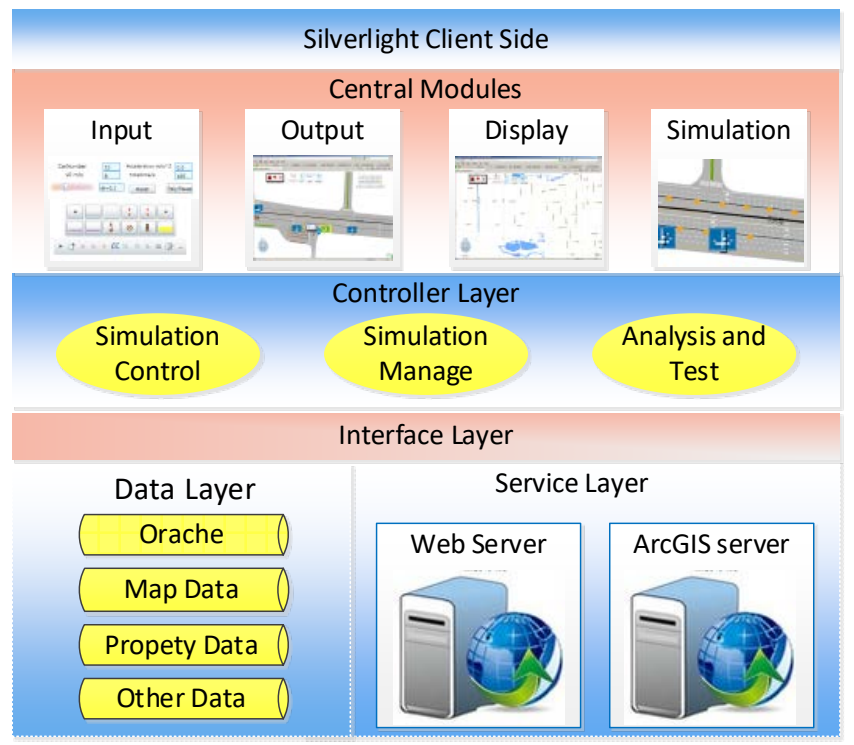

Fig 2. The system framework of micro traffic simulator

The input module is the base of the system and it comprises two parts, one of which is basic road information and the other is simulation parameters setting. As we all known, the basic road information such as the number of lane and lane width is necessary for most classical traffic simulation softwares to build the road network model. It is need to spend much time on making survey of simulation site and creating the road network by hand. However, this simulator proposed in this paper combines with the Web GIS to get the real road basic information and display them by the Silverlight technology. The road network model can be built automatically and the resolution of model can also be improved much. Based on the need of user, the simulation parameter setting part is to make sure the size of simulation area, simulation period, simulation speed, traffic volume, the 
initial speed of head-car, acceleration and so on. These simulation parameters also have the default values.

Output module includes simulation image output and evaluation files output. The simulation image output can display the position of vehicles in real time through the visual interface. The evaluation files output can record the dynamic change of vehicles such as position, speed, acceleration and so on. The evaluation files can be used to calculate the complex traffic operating indexes such as saturation and delay which can reflect the traffic operating condition.

The display module is primarily responsible for dynamic display of GIS map. The main function of this module contains map roaming, zooming, search, location, geometry service, geo-processing and so on.

The simulation module is the most important component of the traffic simulator to describe the spatial position of vehicles, the relationship between the vehicles and the like. This module contains generated model of the vehicle, car-following model $[9,10]$, lane changing model $[11,12]$, free travel model, velocity distribution model.

\section{Case Study}

In order to verify the simulation reasonability and resolution of the microscopic traffic simulator, the cross of Aerospace Bridge road and Fucheng road in Beijing is selected as the simulation area and shown in Figure 3. The total number of input vehicles is fifty thousands and the simulation period is set to be ten thousands seconds. The road section about two kilometers away from the cross in the Fucheng road is selected as an observation point.

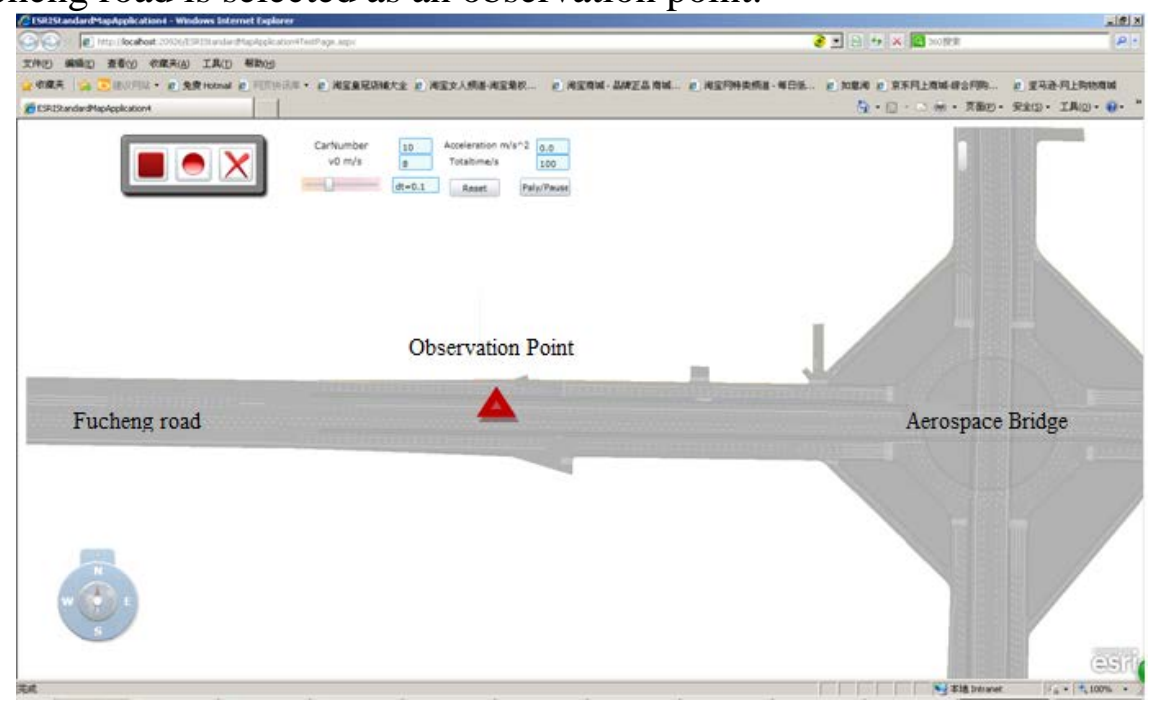

Fig 3. The cross of Aerospace Bridge road and Fucheng road in Beijing

\section{Conclusions}

The microscopic traffic simulation system based on the technologies of Silverlight and Web GIS has the friendly user interface, good interactive experience and the simple Web GIS service invocation model. These features make the Web-based traffic simulation has good prospects and development potential. The simulator designed in this paper is now still in the preliminary stages of development. In next step, lots of efforts should be made to optimize the central module - traffic simulation models. Then the simulation resolution will get improved and the process of simulation is more like the actual traffic operation of China.

\section{Acknowledgments}

The authors would like to thank the Technology Plan of Shenzhen (CXZZ20130322101420409). 


\section{References}

[1] Bloomberg L, Dale J. Comparison of VISSIM and CORSIM traffic simulation models on a congested network. Transportation Research Record: Journal of the Transportation Research Board, 2000 (1727): 52-60.

[2] Cameron G D B, Duncan G I D. PARAMICS-Parallel microscopic simulation of road traffic. The Journal of Supercomputing, 1996, 10(1): 25-53.

[3] Byrne J, Heavey C, Byrne P J. A review of Web-based simulation and supporting tools. Simulation modelling practice and theory, 2010, 18(3): 253-276.

[4] Miller J A, Seila A F, Xiang X. The JSIM web-based simulation environment. Future Generation Computer Systems, 2000, 17(2): 119-133.

[5] Kim D H, Kim M S. Web GIS service component based on open environment //Geoscience and Remote Sensing Symposium, 2002. IGARSS'02. 2002 IEEE International. IEEE, 2002, 6: 3346-3348.

[6] Gao S, Qin F, Liu J, et al. Design of the tourism service WebGIS based on Silverlight //2013 21st International Conference on Geoinformatics. IEEE, 2013: 1-5.

[7] Wong W S D, Lee J. Statistical analysis of geographic information with ArcView GIS and ArcGIS. Wiley, 2005.

[8] Loney K. Oracle database 10g: the complete reference. McGraw-Hill/Osborne, 2004.

[9] Gipps P G. A behavioural car-following model for computer simulation. Transportation Research Part B: Methodological, 1981, 15(2): 105-111.

[10] Benekohal R F, Treiterer J. CARSIM: Car-following model for simulation of traffic in normal and stop-and-go conditions. Transportation research record, 1988 (1194).

[11] Kesting A, Treiber M, Helbing D. General lane-changing model MOBIL for car-following models. Transportation Research Record: Journal of the Transportation Research Board, 2007.

[12] Gipps P G. A model for the structure of lane-changing decisions. Transportation Research Part B: Methodological, 1986, 20(5): 403-414. 